\title{
Quality Counts: Behavioral Health Care Services with Youth Should be guided by Outcome Metrics
}

\author{
Robert D Friedberg, Erica Rozbruch M
}

Center for the Study and Treatment of Anxious Youth at Palo Alto University

*Corresponding author: Robert D Friedberg, Center for the Study and Treatment of Anxious Youth at Palo Alto University, USA, Tel: 650-961-9300 x3617; E- mail: rfriedberg@paloaltou.edu

Rec date: Feb 1, 2016; Acc date: Feb 3, 2016; Pub date: Feb 5, 2016

Copyright: (C2016 Robert D Friedberg This is an open-access article distributed under the terms of the Creative Commons Attribution License, which permits unrestricted use, distribution, and reproduction in any medium, provided the original author and source are credited.

\section{Editorial}

The Affordable and Accountable Care Act (ACA) challenges psychiatrists, psychologists, and other behavioral health care providers in the United States to "step up their game." The behavioral health care ecosystem is changing from an environment that in the past emphasized volume of care to now one that is driven by value-added benefits. Clinical accountability will be prized and behavioral health care providers are well-advised to develop ways to transparently demonstrate good clinical outcomes.

The pivotal task for those who provide and manage psychiatric services is not whether to monitor outcomes but how to account for quality services. Measurement-based practice transcends mere pre-post testing of treatment effects. Rather, measurement based clinical work requires regular repeated monitoring over time with real-time feedback delivered to providers and patients [1]. The advantages of meaningful outcome measurement are well- recognized. Clinicians who closely monitor their patients' treatment outcome on a session-to-session basis receive immediate feedback about treatment gains, losses and ties. In this way, clinical decision making is driven by individualized data collection and analysis [2]. Providers and patients alike are able to see what are linked to spirited progress, stalled improvement, and deteriorating functioning. Sharing progress data with patients and families facilitates greater treatment engagement and insures informed consent throughout the course of treatment. Fortunately, there are a number of nomothetic and idiographic measurement options for tracking clinical outcomes. While some well-known progress tracking instruments are relatively costly such as the Children's Depression Inventory, Beck Depression Inventory, and Conner's Rating Scales, other nomothetic tools are inexpensive. Most recently, Beidas [3] listed various free and reliable nomothetic measures for treatment monitoring. Instruments should be brief, psychometrically solid, responsive to change, and linked to patients' goals for care.

Clinical dashboards are data management tools that guide provider decision making. Robust clinical dashboards include information on treatment progress as well therapeutic processes. Contextualized Feedback Intervention and Training provides state of the art tools for clinical measurement [4].
CFIT is rooted in several theories of change and contains multiple assessment measures which assess both treatment progress as well as treatment processes.

The Managing and Adapting Practice platform (MAP) [5] enables clinicians to build customized evidence-based treatment packages for individual patients. MAP incorporates the well-studied and highly regarded Modular Approach to Therapy with Children with Anxiety, Depression, Trauma, and Conduct problems (MATCH) [6].MAP incorporates selfcontained progress metrics and is flexible enough to allow clinicians to add their own instruments to the measurement mix.

Functional improvements are the most powerful and compelling indicators of change. Quality of life indices are quite useful in this regard. However, providers might measure more personalized functional markers such as number of days missed at school, decreased suspensions, and reductions in medication dosages.

Regardless of the measurement procedures and processes used, the time has come for greater accountability and measurement-based clinical practice. Frankly, the demand for greater quality is overdue! Meaningful measurement of clinical effectiveness propels our profession forward. First and most importantly, patient care is improved. Second, outcome metrics demonstrating effective care documents behavioral health services' value-added benefits to individual patients and the general public. Finally, measurement based care is most likely to yield greater reimbursements for psychiatric services. Quality counts; so let's embrace measurement based psychiatric practice [7-9].

\section{References}

1. Kelley SD, Bickman L (2009) Beyond outcome monitoring: Measurement Feedback Systems in child and adolescent clinical practice. Curr Opin Psychiatry 22: 363-368.

2. Garland AF, Bickman L, Chorpita BF (2010) Change what? Identifying quality improvement targets by investigating usual mental health care. Adm Pol Mental HIth \& Mental HIth Srvs, 37: 15-26. 
3. Beidas RS, Stewart RE, Walsh L, Lucas S, Downey MM, et al. (2015) Free, brief, and validated: Standardized instruments for low resource mental health settings. Cog Beh Pract 22: 5-19.

4. Bickman L (2008) A measurement feedback system (MFS) is necessary to improve mental health outcome. Jrnl Am Acad Child Adol Psych 47: 1114-1119.

5. Chorpita BF, Daleiden EL (2014) Structuring the collaboration of science and service in pursuit of a shared vision. Jrnl Clin Child Adol Psy 43: 323-338.

6. Chorpita BF, Weisz JR (2009) Modular Approach to Therapy with Children with Anxiety, Depression, Trauma, and Conduct Problems. Satellite Beach, Fla: Practice wise pp267.
7. Janicke DM, Fritz AM, Rozensky RH (2015) Healthcare reform and preparing the future child and adolescent psychology workforce. Jrnl Clin Child Adol Psy 44: 1030-1039.

8. Asarnow JR, Hoagwood KE, Stancin T, Lochman J, Hughes JL, et al. (2015) Psychological science and innovative strategies for informing health care redesign: A policy brief. J of Clinical Child and Adolescent Psychology 44: 923-932.

9. Kazdin AE (2008) Evidence-based treatment and practice: New opportunities to bridge clinical research and practice enhance the knowledge base, and improve patient care. American Psychologist 63: 146-159. 